\title{
Assessment of Obstetric and Neonatal Health Services in Developing Country Health Facilities
}

\author{
Albert Manasyan, MD ${ }^{1,2}$, Sarah Saleem ${ }^{3}$, Marion Koso-Thomas ${ }^{4}$, Fernando Althabe ${ }^{5}$, \\ Omrana Pasha, MD ${ }^{3}$, Elwyn Chomba, MD ${ }^{1,2,6}$, Shivaprasad S. Goudar, MD,MHPE ${ }^{7}$, Archana \\ Patel, MD ${ }^{8}$, Fabian Esamai, MD ${ }^{9}$, Ana Garces, MD ${ }^{10}$, Bhala Kodkany, MD ${ }^{11}$, Jose Belizan, \\ MD $^{5}$, Elizabeth M. McClure, MEd ${ }^{12}$, Richard J. Derman, MD ${ }^{13}$, Patricia Hibberd, MD ${ }^{14}$, \\ Edward A. Liechty, MD ${ }^{15}$, K. Michael Hambidge, MD ${ }^{16}$, Waldemar A. Carlo, MD ${ }^{1}$, Pierre \\ Buekens, MD ${ }^{17}$, Janet Moore, MA ${ }^{12}$, Linda L. Wright, MD ${ }^{18}$, Robert L. Goldenberg, MD ${ }^{19}$, \\ and for the EmONC Trial Group
}

${ }^{1}$ Centre for Infectious Disease Zambia, Lusaka, Zambia ${ }^{2}$ Department of Pediatrics, University of Alabama at Birmingham, Birmingham, Alabama ${ }^{3}$ Department of Community Health Sciences, Aga Khan University, Karachi, Pakistan ${ }^{4}$ Perinatology and Pregnancy Branch, Eunice Kennedy Shriver National Institute of Child Health and Human Development, Bethesda, Maryland ${ }^{5}$ Department of Mother and Child Health Research, Institute of Clinical Effectiveness and Health Policy, Buenos Aires, Argentina ${ }^{6}$ Department of Pediatrics, University of Zambia, Lusaka, Zambia ${ }^{7}$ Department of Physiology, KLE's Jawaharlal Nehru Medical College, Belgaum, India ${ }^{8}$ Clinical Epidemiology Unit, Indira Gandhi Government Medical College, Nagpur, India ${ }^{9}$ Department of Pediatrics, Moi University, Eldoret, Kenya ${ }^{10}$ IMSALUD, Francisco Marroquin University, Guatemala City, Guatemala ${ }^{11}$ Department of Obstetrics, KLE's Jawaharlal Nehru Medical College, Belgaum, India ${ }^{12}$ Social, Statistical and Environmental Sciences, Research Triangle Institute, Durham, North Carolina ${ }^{13}$ Department of Obstetrics, Christiana Health Care, Newark, Delaware ${ }^{14}$ Department of Pediatrics, Indiana University, Indianapolis, Indiana ${ }^{15}$ Department of Pediatrics, Massachusetts General Hospital for Children, Boston, Massachusetts ${ }^{16}$ Department of Pediatrics, University of Colorado, Denver ${ }^{17}$ Tulane School of Public Health and Tropical Medicine, New Orleans, LA ${ }^{18}$ Center for Research for Mothers and Children, Eunice Kennedy Shriver National Institute of Child Health and Human Development, Bethesda, Maryland

${ }^{19}$ Department of Obstetrics and Gynecology, Columbia University, New York, New York

\section{Abstract}

Objective-To describe the staffing and availability of medical equipment and medications and the performance of procedures at health facilities providing maternal and neonatal care at African, Asian, and Latin American sites participating in a multicenter trial to improve emergency obstetric/neonatal care in communities with high maternal and perinatal mortality.

Study Design-In 2009, prior to intervention, we surveyed 136 hospitals and 228 clinics in 7 sites in Africa, Asia, and Latin America regarding staffing, availability of equipment/medications, and procedures including cesarean section.

Results-The coverage of physicians and nurses/midwives was poor in Africa and Latin America. In Africa, only 20\% of hospitals had full-time physicians. Only $70 \%$ of hospitals in Africa and Asia had performed cesarean sections in the last 6 months. Oxygen was unavailable in

Address for correspondence and reprint requests Elizabeth M McClure, Statistics and Epidemiology, Research Triangle Institute, Durham, NC 27709 (mcclure @ rti.org).

The authors declare that they have no conflicts of interest. 
$40 \%$ of African hospitals and $17 \%$ of Asian hospitals. Blood was unavailable in $80 \%$ of African and Asian hospitals.

Conclusions-Assuming that adequate facility services are necessary to improve pregnancy outcomes, it is not surprising that maternal and perinatal mortality rates in the areas surveyed are high. The data presented emphasize that to reduce mortality in these areas, resources that result in improved staffing and sufficient equipment, supplies, and medication, along with training, are required.

\section{Keywords}

emergency obstetric and neonatal care; developing countries; perinatal mortality

Developing countries have the highest burden of maternal, fetal, and neonatal deaths. Although progress is being made toward achieving the Millennium Development Goals 4 and 5 of reducing maternal mortality by three-quarters and under- 5 mortality by two-thirds, respectively, these targets will not likely be met before $2015 .{ }^{1}$

Most of the maternal and fetal deaths and many of the neonatal deaths and long-term childhood neurological disabilities are due to obstetric complications, which include sepsis, hemorrhage, preeclampsia/eclampsia, and prolonged and obstructed labor. ${ }^{2}$ Prediction of these conditions is not always possible and many cannot be prevented. Therefore, access to safe and effective obstetric and neonatal services in case of an emergency is crucial in preventing morbidity and mortality.

The International Federation of Gynecology and Obstetrics defines basic obstetric and neonatal services as the availability of antibiotics, oxytocics, anticonvulsants, manual extraction of the placenta, dilation and curettage, assisted vaginal delivery and ability to perform basic neonatal resuscitation (e.g., with bag and mask). ${ }^{3}$ Comprehensive emergency obstetric care includes the availability of the components of basic care as well as cesarean delivery (CS) and blood transfusion capability.

To test the hypothesis that a package of care comprising community mobilization, teaching of home-based lifesaving skills to communities, and enhancing access to upgraded health facilities will improve neonatal and maternal pregnancy outcomes, the Global Network for Women's and Children's Health Research (Global Network) has undertaken a clusterrandomized clinical trial of Emergency Obstetric and Neonatal Care (EmONC). ${ }^{4}$ To better understand the availability of obstetric and neonatal health services within its clusters, prior to the study, we surveyed each of the health facilities in the trial sites and that data are presented in this report.

\section{Methods}

The Global Network EmONC trial, a cluster randomized control trial, is being conducted in 106 clusters in 6 countries (Kenya, Zambia, India - two sites, Pakistan, Guatemala, and Argentina). ${ }^{4}$ A cluster is defined as a contiguous geographic catchment area with at least 300 deliveries per year. All health clinics within the clusters were in rural or semiurban settings except those in Argentina, which were urban. In preparation for the trial, site investigators identified all health facilities serving women located within the study clusters whether public or private. Trained health providers conducted a survey of each of these hospitals and health clinics to assess the staffing level, equipment and supply availability, and medical procedures as well as the communication practices prior to initiation of the trial. Some questions (i.e., equipment, staffing) were completed only through direct observation to ensure the quality of data. The baseline facility assessments were conducted prior to the 
implementation of the interventions. For simplification of presentation, after providing descriptive information for each of the sites, we grouped the sites by continent. For the purpose of reporting survey results, each facility was self-identified as hospital or health clinic by the facilities themselves.

The study, including the survey results reported here, was approved by institutional review boards for every international study site, the United States university counterparts, and the data center at Research Triangle Institute International. The sites completed forms that provided information on availability of specific procedures, equipment, supplies, and communication devices at the facility. Items and activities were recorded as available always, sometimes, or never. The percent of hospitals and health centers with a specific health service available were computed for each site.

\section{Results}

Descriptions of the geographic areas in which the surveys were conducted, the number of facilities surveyed, and the total number of deliveries for 2010 are shown in Table 1.

Altogether, 364 facilities were included in the initial survey, 136 hospitals and 228 health clinics. Data for the delivery rates in Table 1 were derived from the Maternal Newborn Health Registry, a population-based observational study using prospective pregnancy-related data for more than 100 clusters across the sites. Maternal and newborn data were collected during antenatal care visits, at delivery, and at 42 days postdelivery. ${ }^{5}$ Across all the sites, the mean percent of deliveries occurring in a facility was $66 \%$, with a range from $99 \%$ in Argentina to $30 \%$ in Guatemala. Sites in Africa had the lowest percentage of hospital deliveries compared with sites in Asia and Latin America. In the sites in Africa and Asia between $24 \%$ and $45 \%$ of the deliveries occurred in health clinics. Sites in Latin America had very few deliveries in health clinics.

\section{Birth Attendants}

There was a wide range of physician and nurse staffing at the various sites (Table 2). In the African hospitals, physicians were always available in $20 \%$ of the hospitals and never available in $20 \%$. In the Asian hospitals physicians were always available in $91 \%$ of the hospitals and never available in 2\%. In Latin American hospitals, physicians were always available in $44 \%$ of the hospitals and never available in $0 \%$. There was also wide variability in nurse and midwife hospital coverage. In the hospitals in Africa, a physician, nurse, or midwife was always available in $100 \%$ of the hospitals, in Asia 98\%, and in Latin America $89 \%$. In the health clinics, physician availability was far less than in hospitals, with physicians never available in $76 \%, 40 \%$, and $71 \%$ in the health clinics in Africa, Asia, and Latin America, respectively. Nurse and midwife clinic coverage was variable across the sites but was generally higher in Africa and Asia than in Latin America.

\section{Equipment and Essential Medications}

Availability of medical supplies varied in the hospitals and health clinics (Table 3). Anesthesia equipment, which is necessary for lifesaving surgery, was available in $70 \%$ of the African hospitals, $67 \%$ of the Asian hospitals, and $100 \%$ of the Latin American hospitals. Anesthesia equipment was available in $4 \%, 7 \%$, and $4 \%$ in African, Asian, and Latin American health clinics, respectively. Oxygen was available at $60 \%$ of the African, $83 \%$ of the Asian, and $89 \%$ of the Latin American hospitals. Oxygen availability ranged from 8\% in the Latin American clinics to 31\% of the Asian clinics. Blood pressure cuffs, which are crucial to diagnose preeclampsia, were available in at least $78 \%$ of facilities at all sites with the lowest percentages in Africa. Bag and masks for newborn resuscitation were 
available in $90 \%$ of the hospitals in Africa, $98 \%$ in Asia, and $89 \%$ in Latin America. They were also available in $73 \%$ of the clinics in Africa and Asia and $33 \%$ of the health clinics in Latin America.

Table 4 shows reported medication availability by region and type of facility. Antibiotics were not available in 11\% of African hospitals and 32\% of African clinics. Magnesium sulfate, a drug needed to prevent and to treat eclamptic seizures, was available in $70 \%$ of the African and in only $52 \%$ of the Asian hospitals. It was available in far fewer of the clinics. Most of the other medications were variably available with fewer of the clinics than hospitals having specific medications. Blood products had the lowest availability in hospitals and health clinics in all three continents. Availability was 20\%, 18\%, and $33 \%$ in African, Asian, and Latin American hospitals and 19\%, 11\%, and $4 \%$ in clinics in those locations, respectively.

Table 5 summarizes the percent of participating facilities in each region that reported performing a procedure at least once in the 6 months preceding the survey. In Africa and Asia approximately $70 \%$ of the hospitals had performed a CS and dilation and curettage in the last 6 months, and in Latin America, 100\% of the hospitals did so. Use of forceps or vacuum extractors was less common, with $20 \%, 54 \%$, and $22 \%$ of hospitals in Africa, Asia, and Latin America reporting their use in the last 6 months. These procedures were performed far less commonly in the health clinics with no use reported in Latin American health clinics.

\section{Communication}

Finally, we evaluated the presence of communication tools (Table 6). Telephone lines were generally available in hospitals but were far less available in health clinics. Availability of cell phones was near $100 \%$ in hospitals but less so in the clinics. Radio communication was available in about $25 \%$ of hospitals in all locations, but was almost never available in health clinics. Computer/Internet availability was relatively high in the African and Latin American hospitals, less so in Asian hospitals, but rare in health clinics in all locations.

\section{Discussion}

In many developing countries, maternal, fetal, and neonatal mortality rates remain unacceptably high. Most of these deaths are related to the events that occur around labor and delivery, and many could be prevented if the elements of basic and/or emergency obstetric and neonatal care were available. ${ }^{6-8}$

This study, which evaluated hospital and health clinic staffing, the availability of equipment and supplies, medications, communications equipment, and the performance of some key medical procedures, helps explain some of the very high rates of adverse pregnancy outcomes in some low-income countries. First, the availability of key personnel such as physicians, nurses, and midwives is poor in hospitals and worse in clinics. Anesthesia equipment was available in only about $70 \%$ of hospitals and approximately $5 \%$ of clinics in Africa and Asia. Other important lifesaving diagnostic and treatment interventions such as blood pressure cuffs, bag and masks for neonatal resuscitation, and oxygen were far from universally available. Blood products were available in only $20 \%$ of African and Asian hospitals, areas where hemorrhage is the major cause of maternal death. Availability of magnesium sulfate, uterotonics, and antibiotics, essential medications for emergency obstetric care, was also very low. The fact that CS was performed in only $70 \%$ of the African and Asian hospitals, and in less than 5\% of the clinics, confirms that this important intervention is often not available to women and fetuses in need. Without staff with 
equipment able to perform key procedures around the clock, 7 days a week, women, fetuses, and newborns will die unnecessarily. ${ }^{9,10}$

A limitation of the study was that the availability of equipment, supplies, and medication was recorded but actual use was not measured. We are aware of many cases in developing countries where various technologies are available but not actually used. We are also aware that various medications are in stock, but used either incorrectly or not at all. We also did not assess the skills of the health care providers. Not all physicians can perform a CS or perform one competently. Knowledge of the capabilities, knowledge, and skill levels of the providers would be an important addition to the availability of staff and use of equipment, supplies, and medications.

There is a push for more women to deliver in hospitals or health clinics, ${ }^{11}$ but the availability of services in many of those facilities is still severely deficient. Factors such as community involvement and mobilization, access to financial resources, and level of education contribute to utilization of obstetric services. ${ }^{12}$ Although community-based strategies may still have a role in reducing maternal mortality rate to a certain extent in geographic areas with very high mortality rates, ${ }^{13}$ there exists no evidence that a maternal mortality rate below 100 per 100,000 live births can be achieved through community-based strategies alone without access to emergency obstetric care at health facilities. ${ }^{14}$

\section{Conclusion}

Access to reproductive health services including emergency obstetric care is often considered a basic human right, yet neither basic nor comprehensive perinatal services are the norm in most resource-poor settings. ${ }^{9}$ To improve maternal and newborn health care, there is need for more human resources in rural settings and a system to ensure their constant availability parallel with improved services. ${ }^{15,16}$ Coverage of effective interventions is low, however, and many opportunities are missed to provide quality care within existing health systems. There is a growing consensus that building stronger health systems is vital to achieving better perinatal health outcomes, especially in countries where the health outcomes are very poor.

\section{Acknowledgments}

This study was funded by grants from the Eunice Kennedy Shriver National Institute of Child Health and Human Development (U01 HD040477, U01 HD043464, U01 HD040657, U01 HD042372, U01 HD040607, U01 HD058322, U01 HD058326, and U01 HD040636).

\section{References}

1. Islam M, Yoshida S. MDG 5: how close are we to success? BJOG. 2009; 116(Suppl 1):2-5. [PubMed: 19740160]

2. Khan KS, Wojdyla D, Say L, Gülmezoglu AM, Van Look PF. WHO analysis of causes of maternal death: a systematic review. Lancet. 2006; 367:1066-1074. [PubMed: 16581405]

3. FIGO Committee Report. Essential interventions for maternity care in low resource countries. Int J Gynaecol Obstet. 2009; 104:80.

4. Pasha O, Goldenberg RL, McClure EM, et al. Communities, birth attendants and health facilities: a continuum of emergency maternal and newborn care (the global network's EmONC trial). BMC Pregnancy Childbirth. 2010; 10:82. [PubMed: 21156060]

5. McClure EM, Pasha O, Goudar SS, et al. Global Network Investigators. Epidemiology of stillbirth in low-middle income countries: a Global Network Study. Acta Obstet Gynecol Scand. 2011; 90:1379-1385. [PubMed: 21916854] 
6. Paxton A, Maine D, Freedman L, Fry D, Lobis S. The evidence for emergency obstetric care. Int J Gynaecol Obstet. 2005; 88:181-193. [PubMed: 15694106]

7. McClure EM, Goldenberg RL, Bann CM. Maternal mortality, still-birth and measures of obstetric care in developing and developed countries. Int J Gynaecol Obstet. 2007; 96:139-146. [PubMed: 17274999]

8. AbouZahr C, Wardlaw T. Maternal mortality at the end of a decade: signs of progress? Bull World Health Organ. 2001; 79:561-568. [PubMed: 11436479]

9. Casey SE, Mitchell KT, Amisi IM, et al. Use of facility assessment data to improve reproductive health service delivery in the Democratic Republic of the Congo. Confl. Health. 2009; 3:12. [PubMed: 20025757]

10. Kongnyuy EJ, Hofman J, Mlava G, Mhango C, van den Broek N. Availability, utilisation and quality of basic and comprehensive emergency obstetric care services in Malawi. Matern Child Health J. 2009; 13:687-694. [PubMed: 18581221]

11. Lim SS, Dandona L, Hoisington JA, James SL, Hogan MC, Gakidou E. India's Janani Suraksha Yojana, a conditional cash transfer programme to increase births in health facilities: an impact evaluation. Lancet. 2010; 375:2009-2023. [PubMed: 20569841]

12. Bhutta ZA, Darmstadt GL, Haws RA, Yakoob MY, Lawn JE. Delivering interventions to reduce the global burden of stillbirths: improving service supply and community demand. BMC Pregnancy Childbirth. 2009; 9(Suppl 1):S7. [PubMed: 19426470]

13. Anwar I, Kalim N, Koblinsky M. Quality of obstetric care in public-sector facilities and constraints to implementing emergency obstetric care services: evidence from high- and low-performing districts of Bangladesh. J Health Popul Nutr. 2009; 27:139-155. [PubMed: 19489412]

14. Koblinsky MA, Campbell O, Heichelheim J. Organizing delivery care: what works for safe motherhood? Bull World Health Organ. 1999; 77:399-406. [PubMed: 10361757]

15. World Health Organization. Geneva, Switzerland: World Health Organization; 2005. The World Health Report 2005. Make Every Mother and Child Count; p. 219

16. World Health Organization. Geneva, Switzerland: World Health Organization; 2006. The World Health Report 2006: Working Together for Health; p. 209 


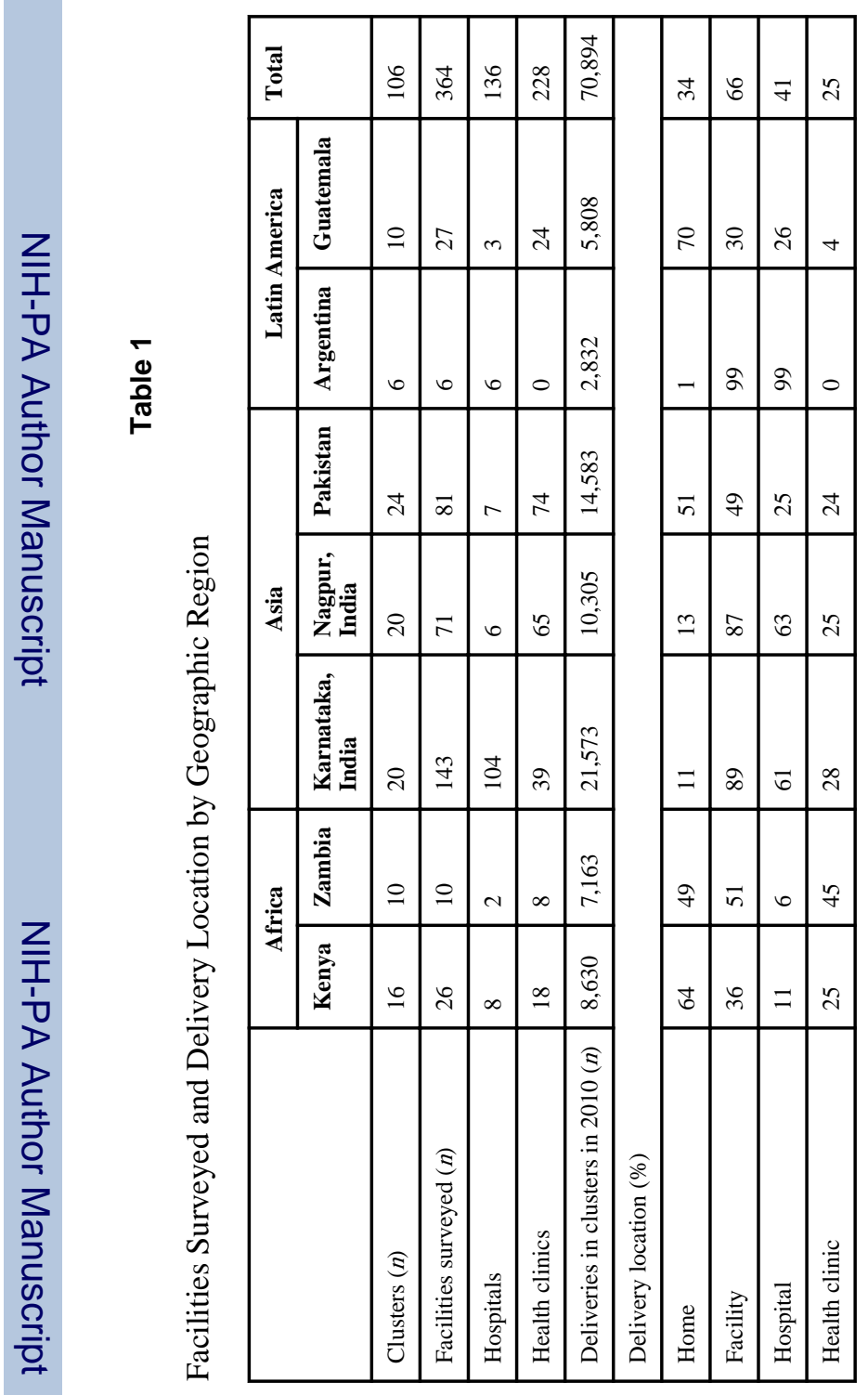

Am J Perinatol. Author manuscript; available in PMC 2013 October 01. 


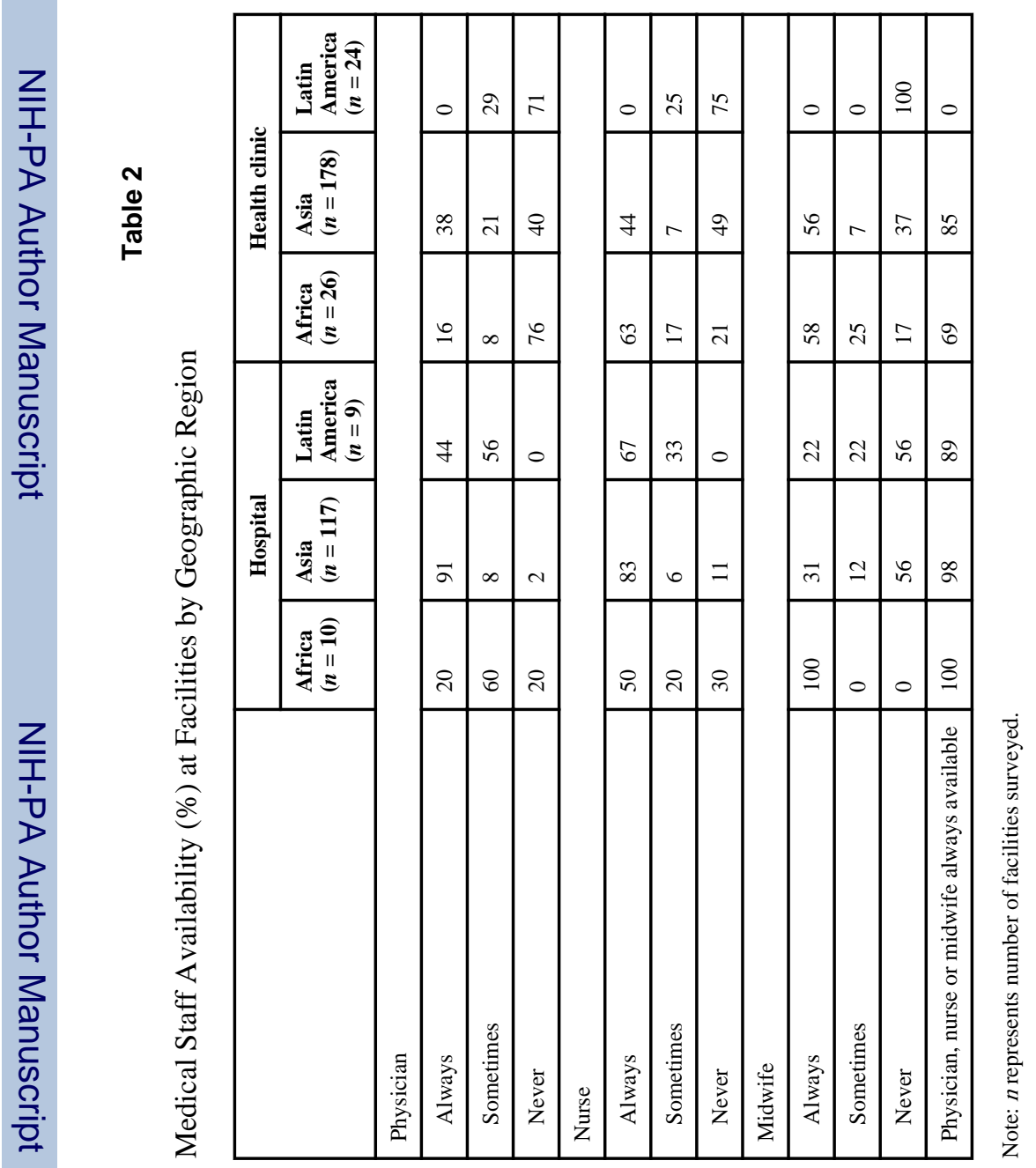

Am J Perinatol. Author manuscript; available in PMC 2013 October 01. 


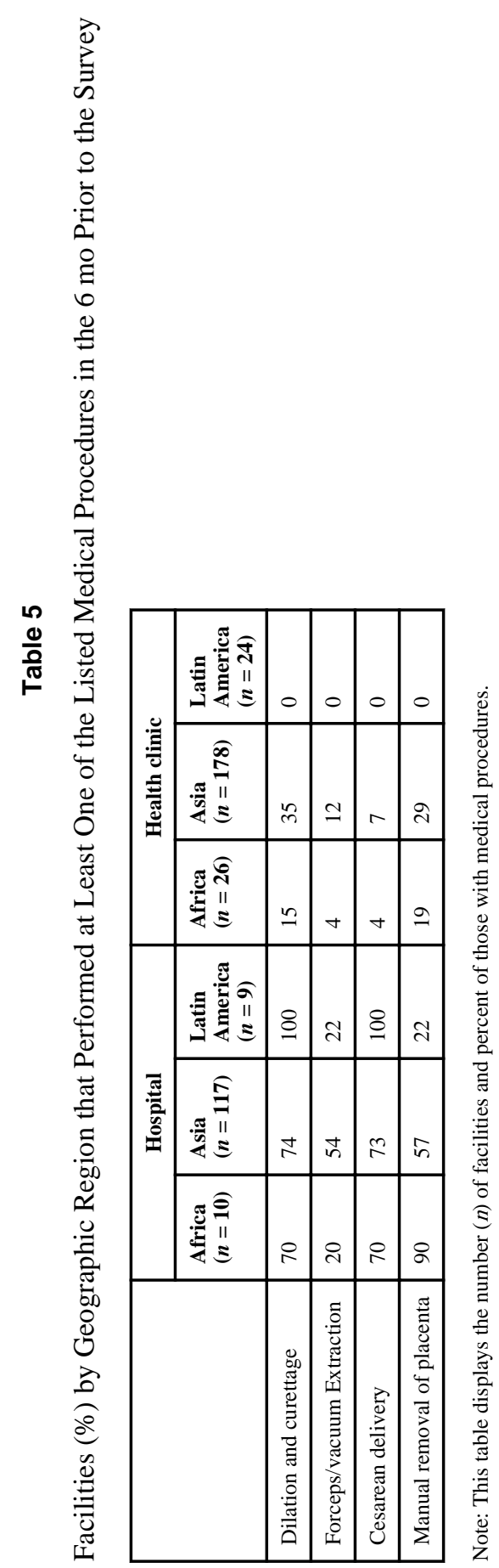

Am J Perinatol. Author manuscript; available in PMC 2013 October 01. 


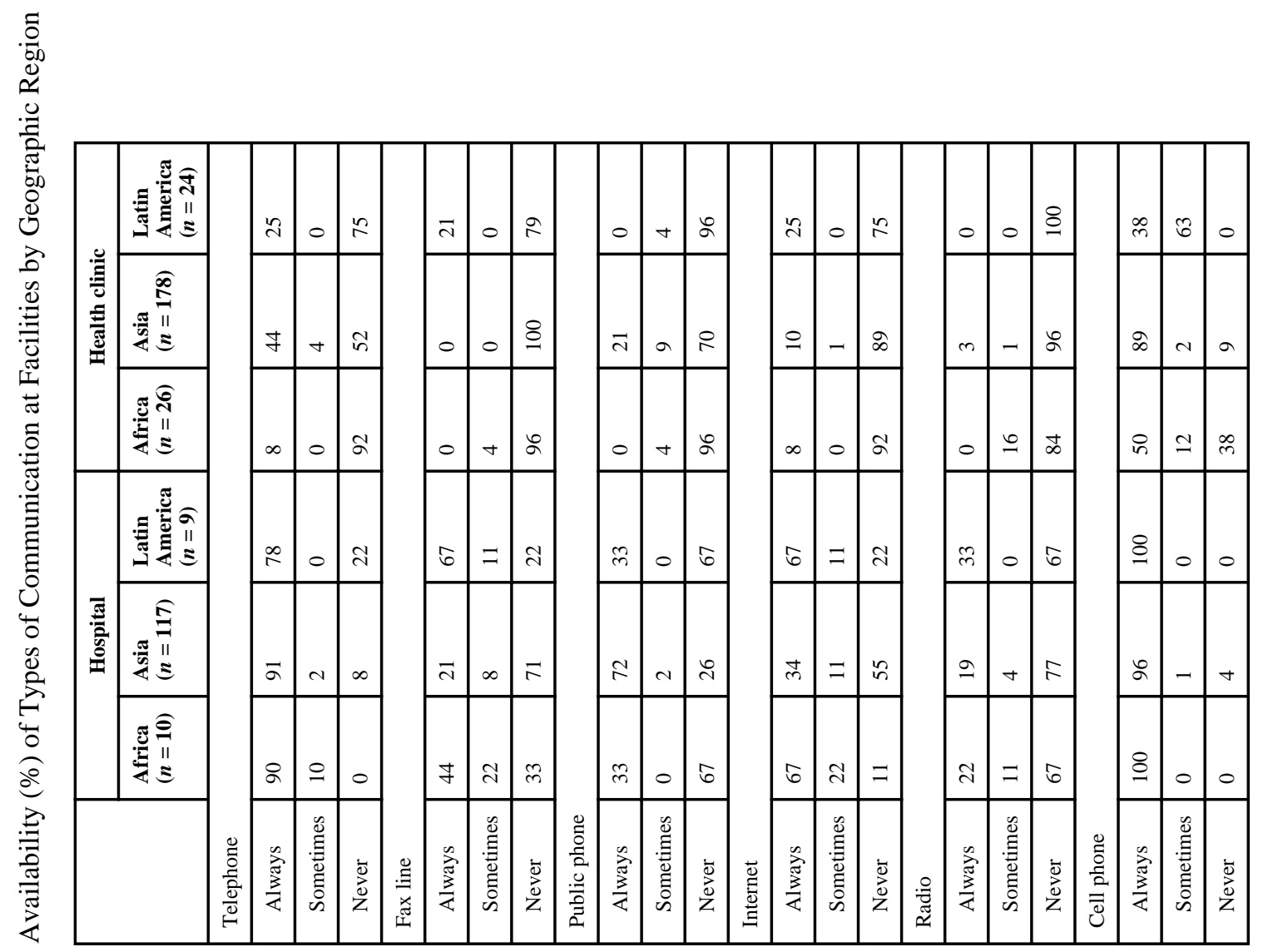

